\title{
La autonomía del derecho sanitario en la organización institucional de las provincias argentinas
}

\author{
The autonomy of health law in the institutional organization of argentinian provinces
}

A autonomia da lei de saúde na organização institucional das províncias argentinas

RESUMEN: Una vez planteada la autonomía del derecho sanitario en la Argentina, a partir de sentar sus bases, se observará la relación a través de los distintos niveles de gobierno, ya que la existencia de un Estado Federal en la Argentina se traduce en un sistema jurídico multinivel y plurilegislativo en el cual coexisten diversos ordenamientos jurídicos. Por ello resulta necesario conocer el ámbito de competencia específico de cada orden de gobierno y que asuma fundamental importancia la articulación y cooperación en las relaciones intergubernamentales, lo que no está exento de algunas dificultades: ¿En qué consisten los deberes positivos de los distintos órdenes de gobierno, ¿cuál es su alcance y quiénes responden por su cumplimiento? Así como la doctrina ha trabajado muchas cuestiones atinentes a la organización institucional de las provincias argentinas, ha faltado, a nuestro criterio, un estudio transversal en relación con una rama del derecho sumamente importante: el derecho sanitario.

Palabras Ilave: Derecho Sanitario. Legislación Sanitaria. Derecho a la Salud.

ABSTRACT : We will consider the autonomy of sanitary law in Argentina, in order to establish its bases, to observe the relationship through different levels of government, since the existence of a Federal State in Argentina that implies a Multilevel and multiregulated legal system. Therefore, it is necessary to know the specific field of competence of each order of government and the articulation and cooperation in intergovernmental relations, which implies some difficulties: What are the responsibilities of the different orders of government? What are the accountable for compliance? The doctrine has dealt with many questions concerning the institutional organization of Argentina, but it has been lacking a study about a very important branch of the law: sanitary law.

Keywords: Health law. Health Legislation. Right to Health

RESUMO: Uma vez levantada a autonomia do direito sanitário na Argentina para, a partir de suas bases fundamentais, se observará a relação por meio de distintos níveis do

\footnotetext{
1 Abogado, Especialista en Gestión Administrativa de los Servicios de Salud, Posgraduado en Derecho Comunitario, Políticas Públicas y Gestión de Riesgos. Profesor de la Universidad Nacional de Córdoba y de la Universidad Blas Pascal. Prosecretario Legislativo del Poder Legislativo de la Provincia de Córdoba. Córdoba, Argentina. E-mail: joseemilioortega@hotmail.com

${ }_{2}$ Abogado, Magíster en Estudios Internacionales. Docente en la Universidad Nacional de Córdoba. Director de Derechos de Aterrizaje y Autorizaciones del Ministerio de Comunicaciones de la Nación. Córdoba, Argentina. E-mail: santiagomesposito@gmail.com
} 
governo, já que a existência do Estado Federal na Argentina se traduz no sistema jurídico multinível e plurilegislativo no qual coexistem diversos ordenamento jurídicos. Por ele resulta necessário conhecer o âmbito de competência específico de cada ordem do governo e que assuma a fundamental importância da articulação e cooperação das relações intergovernamentais, que não está isento de dificuldades: Em que consistem os deveres positivos das distintas ordens do governo, qual é o seu alcance e quem responde por seu cumprimento? Assim como a doutrina tem trabalhado muitas questões atinentes à organização institucional das províncias argentinas, tem faltado, a nosso critério, um estudo transversal na relação com um ramo do direito sumamente importante: o direito sanitário.

Palavras chave: Direito sanitário. Legislação sanitária. Direito à saúde.

\section{Presentación del tema}

Cualquier cruce que se ensaye, sobre derecho y salud, nos lleva naturalmente a un primer problema, de orden terminológico.

Carece de sentido repetir lo que otros estudios han explicado y desarrollado en extenso, respecto a la polisemia de ambos términos. Como sabemos, "derecho" denota norma, facultad y también "corpus" científico. Mientas que "salud", definida globalmente como "el estado de completo bienestar físico, mental y social, y no solamente la ausencia de afecciones o enfermedades" (1), se proyecta, como aquella a innumerables aspectos de la vida en relación, que exceden ampliamente el marco de profesiones o actividades y el sistema institucional estructurado para acercar la oferta y la demanda pública o privada de prestaciones. Así, en la Conferencia Internacional sobre la Promoción de la Salud reunida en Ottawa (2), se expresó que "la salud no concierne exclusivamente al sector sanitario", concretando una serie de afirmaciones que desvincula a la salud de la órbita de los procesos clásicos de salud-enfermedad-atención relacionándola a esferas más vastas.

La compleja relación entre hombre-sociedad-cultura y derecho, que se proyecta tanto en plano estrictamente normativo y como en la dimensión axiológica, es lo que define al mundo jurídico y al derecho en particular como un fenómeno "multidimensional", aquel que enseña Martinez Paz (3) en el que los fenómenos jurídicos están conformados por la relación "hombre-sociedad-cultura-derecho" en el que el hombre vive la realidad como realidad humana, social, cultural y jurídica. A su vez, procesos sociales "transversales" y transformadores, como la globalización, han generado profundos cambios en la identidad 
individual y colectiva, otorgando a los habitantes del planeta una mayor conciencia sobre éste: como se ha dicho, conciencia de ser ciudadanos del mundo, en una dinámica mediada por las tecnologías de la información y comunicación (4).

En ese mundo tan diverso, tan dinámico, pero también más conectado, surge la necesidad de afirmar algunos conceptos esenciales para luego aprehenderlos en la multiplicidad o la "multidimensional". Al hablar de derecho y salud, podemos encarar dos caminos. Si "salud en un sentido más laxo y más general, más relacionado con la etimología salus -que recogía la idea de salvatio, en el uso de la "v" que hacía el latínpodríamos estar efectuando un examen del ordenamiento jurídico o del sistema jurídico o del complejo jurídico que incluya también el estado de la ciencia. Un análisis de cuán fuerte, organizado, eficaz o eficiente se encuentra el orden de que se trate. Algo así como "La salud del derecho -norma particular, facultad, ordenamiento, sistema, disciplina jurídica etc.-" o "-si llegásemos a esa condición- "un derecho que goza de buena salud".

O en su defecto, podemos estar refiriéndonos a otra vinculación del derecho con la salud; aquella que relaciona a las normas, las facultades y las disciplinas jurídicas, con el "campo de la salud", el cual, como explicaba Lalonde (5) es muy difícil de delimitar conceptualmente y que él proponía desagregar en "cuatro amplios componentes": biología humana, medio ambiente, estilo de vida y organización de la atención de la salud.

La amplitud del concepto requiere la necesidad de delimitarlo. Estamos de acuerdo con relacionarlo con el bienestar, el contexto ambiental y la calidad de vida, pero resta reflexionar hasta qué punto será posible tornar operativos los derechos, para lo cual el establecimiento de objetivos y metas es fundamental. Por caso debemos tener en cuenta los determinantes de la salud, es decir, las condiciones en las que las personas nacen, crecen y viven; condiciones que hacen posible vivir con salud, tales como el acceso al agua segura, condiciones adecuadas de vivienda, alimentación, trabajo digno, entre otras. En otras palabras, desde un costado, hay una obligación que va más allá de la protección del enfermo. Desde el otro, no existe un ilimitado derecho a estar sano, sino una garantía de contar con acceso a prestaciones suficientes para procurar un adecuado "estado de salud", en un contexto de permanente evolución sobre esta idea. Entre ambas riberas, y sostenido en un amplio cauce, fluirá un intenso torrente. 
Enseña Martínez Paz (3) que "La delimitación de fronteras implica un principio de autonomía que se traduce en metodologías, lenguajes, técnicas y propuestas". Ciertamente, la transversalidad del Derecho Sanitario hace referencia a su propia complejidad. Efectivamente se complementa con otras ramas para su concreción en el caso, manteniendo siempre presente rasgos que le son propios. El maestro Donati aportaba, que el nacimiento de una disciplina autónoma descansaba en tres pilares: novedad orgánica, especialidad en los principios y tendencia a la completividad. Surge una nueva rama del tronco jurídico, en este caso el "Derecho Sanitario", cuando, por aparición de hechos o situaciones novedosas, que cambian el ritmo de la vida en relación, las especies clásicas no pueden dar suficiente respuesta a todos los requerimientos jurídicos consecuentes, o cuando su desarrollo reciente es tal, que ya no puede ser abordado por una de ellas en particular.

Sin dudas la compleja satisfacción de la salud, como advertimos en su definición tradicional, sus problemas científicos - técnicos - éticos propios y el aspecto antropológico -el hombre -sano o enfermo- y su particular condición y destino en el mundo-, requieren especial tratamiento. Eso es así porque la noción de salud ha ido evolucionando a lo largo de la historia, desde un enfoque médico-biológico hasta un concepto global e integral que incorpora el paradigma socio-ecológico (3).

\section{El derecho de la salud y la legislación sanitária}

En tanto facultad subjetiva o intersubjetiva, el llamado "derecho a la salud" posee rango fundamental, tanto en el plano individual como considerado desde su incidencia colectiva. Se trata de un derecho humano, por estar intrínsecamente "vinculado a la protección del estatuto de persona" (3), recogido en instrumentos internacionales y regionales, que presenta elementos propios o característicos que determinan su indispensable operatividad. Asimismo es doctrina consolidada de la Corte Suprema de Justicia de la Nación que el Estado Nacional está obligado a proteger la salud pública (3) pues

al derecho está comprendido dentro del derecho a la vida, que es el primer derecho natural de la persona humana pre-existente a toda legislación positiva, entendiendo que en el preámbulo de la Constitución ya se encuentran expresiones referidas al bienestar general, objetivo preeminente 
en el que, por cierto, ha de computarse con prioridad indiscutible la preservación de la salud. (2)

La consagración de esa facultad es relativamente reciente. La demanda de bienes sanitarios o asistenciales transitó un largo recorrido hasta su caracterización actual, que naturalmente exige que el Estado le otorgue plena satisfacción, como apunta el fallo de la Corte Suprema citado supra. Como es sabido, históricamente la salud comenzó a demandarse y brindarse dentro del mercado privado, hasta su colectivización en el siglo XIX (caridad - confesional), su posterior secularización (policía de salubridad y servicios asistenciales públicos) y en el marco de una creciente diversificación tras la primera posguerra, una plena asunción, por parte del estado de un complejo de acciones, sustentado en políticas públicas que a su vez fueron consecuencia de una segunda y hasta una tercera generación de derechos constitucionales en el marco del Estado de Bienestar y la organización de un sistema de derecho internacional que asumió capítulos importantes de la agenda pública de la segunda posguerra y propició la globalización de ciertos objetivos y la internalización, por parte de todos los Estados, de la obligación de brindar determinados bienes públicos, entre los cuales claro está, se destacó la salud.

No obstante, esta experiencia se materializó en diferentes modelos (estatales, privados con regulación pública, mixtos, seguridad social, servicio nacional, asistencia pública, etc.). En ese contexto, nuestro sistema de salud se caracteriza por la coexistencia de diversos modelos, que se reporta segmentada y fragmentada. (6)

Esta diversidad puede advertirse en las distintas definiciones de "derecho sanitario" o "derecho de la salud" que se conocen, muchas de ellas relacionadas con el modelo adoptado, y dentro de éste, con el tipo de esquema que sigue ese paradigma, a veces más asociado a la práctica médica, a veces pensado desde una perspectiva más integral o de "equipo de salud", y a veces aún más integrada dentro de un contexto institucionalprestacional-técnico aún más complejo.

Ya hemos dicho que en tanto derecho humano, presenta aptitud para identificar y abordar cuestiones que trascienden la tensión salud-enfermedad, apto para instrumentar políticas públicas relacionada con los componentes del "campo de la salud".

El doctor Ramón Carrillo, primer Ministro de Salud de la República Argentina, anticipándose a su tiempo, empleó por primera vez de modo oficial o institucional el 
concepto "derecho sanitario", promoviendo el dictado de un Código Sanitario, la designación de un juzgado especializado en su aplicación y resaltando la necesidad de plantear los conflictos jurisdiccionales en la materia con las provincias en el Plan Sintético de Salud Pública 1952-1958.

Toda esta dinámica, independientemente del modelo o paradigma utilizado, se materializa en el marco de un sistema de salud, caracterizado por la OMS como "la suma de todas las organizaciones, instituciones y recursos cuyo objetivo principal consiste en mejorar la salud" (7). Este "sistema" demanda, a su vez, innumerables recursos: personal, financiación, información, suministros, transportes y comunicaciones, así como una orientación y una dirección generales. A su vez, está exigido a brindar una oferta razonable mediante prestaciones preventivas o asistenciales -caracterizadas como servicios de consulta, tratamientos, terapias, etcétera- que respondan a las necesidades y posibilidades sociales.

Proyectado este concepto sobre la realidad sanitaria argentina, y teniendo en cuenta la segmentación del sistema aludida más arriba, las políticas públicas en el área de la salud deben lidiar con un doble frente de fragmentación. Por un lado, la que deriva de la organización federal del país, que se refleja en el subsistema público -desagregado en nacional, provincial y municipal- y en las dificultades para coordinar políticas entre las jurisdicciones. Y por otro, la compartimentación entre sectores que caracteriza al sistema: la diferenciación entre los segmentos público, de la seguridad social, fuertemente diversificado a su interior, y un subsector privado de características muy diversas según cada región. (8)

Tras la reforma estructural de los años 90 -privatizaciones, descentralización, desregulación-, que impactó notablemente en el campo de la salud, este proceso de fragmentación se agudizó. Señala Garay (9) que el objeto capital de regulación de la legislación sanitaria en los años 90 era el "mercado" y la normativa que lo caracterizó, fue la que desreguló el sistema nacional de obras sociales. No obstante, planteando un estándar, piso o umbral: el Programa Médico Obligatorio (PMO), creado en 1995.

Tras la crisis de ese modelo, como consecuencia de problemas más profundos experimentados por la sociedad y las instituciones argentinas y tras los dramáticos sucesos de 2001, la presidencia provisional de Duhalde decretó la emergencia sanitaria 
nacional -durante 2002- aún vigente. Una serie de procesos de mediación entre la dirigencia política y la sociedad civil organizada -en particular de la Iglesia-, cimentó consensos que influyeron positivamente en la renovada consideración de los asuntos sanitarios, con la firma de una serie de acuerdos entre Nación y Provincias, que fueron matriz de un nuevo enfoque, en el cual la persona, y no el mercado, recuperaron centralidad en la concepción de políticas públicas para el sector, logrando una trascendencia mayor en la legislación en salud que la había tenido en la década anterior. Se desarrollaron líneas de política en materia de la salud sexual y procreativa (ley 25.673 del Programa nacional de salud sexual y procreación responsable) y también reforzando el protagonismo del Estado Nacional en articulación con las jurisdicciones subnacionales se implementaron políticas dirigidas a garantizar el acceso equitativo a los medicamentos mediante la ley 25.649 de Promoción de la utilización de medicamentos por su nombre genérico. Se implementaron programas y lineamientos de política tendientes a articular intervenciones desde el Estado Nacional, como el Plan Federal de Salud 2004-2007 del Ministro de Salud Ginés González García, en el gobierno del Dr. Néstor Kirchner cuyo fin fue establecer un modelo basado en la estrategia de Atención Primaria de la Salud (APS). Estos nuevos lineamientos buscaron vincular la asignación de recursos al cumplimiento de objetivos y metas cuantificables, por parte de las jurisdicciones. Entre ellos se destaca el Plan Nacer (reformulado en 2012 como Programa Sumar y reforzado por la Asignación Universal por Embarazo), un programa para la atención de la salud materno-infantil que actualmente se extiende a otros segmentos etarios. En 2004 se anunció el Plan Federal de Salud que propuso un modelo sanitario basado en la construcción de redes de atención y fundamentado en la estrategia de atención primaria como organizadora del sistema.

Entre otras leyes se destacan la ley 26.396 de declaración de interés nacional de la obesidad, la anorexia, la bulimia y otros trastornos alimentarios, ley 26.529 de Derechos del Paciente; ley 26.657 de Derecho a la Protección de la Salud Mental; ley 26.862 de acceso integral a los procedimientos y técnicas médico-asistenciales de reproducción médicamente asistida; ley 26.872 de cirugía reconstructiva en patologías mamarias; ley 26.905 de reducción de consumo de sodio; ley 26.687 de regulación de la publicidad, promoción y consumo de los productos elaborados con tabaco; ley 26.689 de promoción del cuidado integral de la salud de las personas con Enfermedades Poco Frecuentes; ley 27.071 cobertura total para las personas ostomizadas; ley 26.928 de creación del sistema 
de protección integral para personas trasplantadas; ley 26.396 de interés nacional la prevención y control de trastornos alimentarios; ley 27.043 de interés nacional el abordaje integral de las personas que presentan trastornos del espectro autista.

Muchas de estas leyes fueron reglamentadas por decretos del Poder Ejecutivo, o directamente instrumentadas por resoluciones de la autoridad de aplicación y otras esperan todavía por que comience su ejecución.

Son innegables los avances de la legislación sanitaria en los últimos trece o catorce años. Se prevé la cobertura de muchos tratamientos, pero no se establece el origen de los fondos para cubrir las patologías y muchas veces no se determina criterio alguno para la cobertura de las prestaciones. El hecho de no contemplar las fuentes de financiación de las prestaciones que se exigen complica la actividad, no sólo de los efectores de salud, sino también de los organismos regulatorios. Se observa una total independencia entre la prestación y la financiación.

Asimismo, en el área de la seguridad social en salud no se introdujeron cambios significativos, y la complejidad política e institucional del sistema no alentó iniciativas dirigidas a fortalecer tendencias hacia la unificación y coherencia de un sistema de salud, insistimos, altamente fragmentado.

Por otra parte, dada la estructura federal del país, los gobiernos provinciales cuentan con relativa autonomía en materia de políticas de salud pública y con la mayor parte de responsabilidades en la provisión de servicios, lo que hace que los lineamentos del nivel nacional tengan muchas veces una incidencia "mediada", sujeta a la incorporación de las normas nacionales a los ordenamientos jurídicos locales, estando la adhesión condicionada a las coincidencias en el plano político más general. A su vez, los municipios tienen a cargo la ejecución de programas y la administración de los servicios de su órbita. Finalmente, en la escena provincial, el fuerte desarrollo de la seguridad social, de características centralizadas en su regulación y contralor, impone a su vez, importantes restricciones a la autonomía real de los gobiernos provinciales en la provisión de salud dentro de sus territorios (10).

Entre las principales funciones de un sistema de salud se consideran: la provisión de servicios, la generación de recursos, la financiación y la gestión. Dado que involucra a todas las organizaciones e instituciones y recursos destinados a "actividades de salud" 
(servicios, iniciativas, etcétera), dentro de un sistema, como hemos dicho, encontramos sectores o "subsistemas", clasificados en razón del origen del financiamiento y la organización. a) público: financiamiento impositivo -y fondos internacionales, que atienen a aproximadamente el $40 \%$ población, a través de más de 1.200 efectores -la mayoría hospitales-, regulado por legislación concurrente; b) Obras Sociales: seguro de salud de aporte obligatorio, entre el 7 y el 12,5, sindicales, provinciales, universitarias, PAMI, todas ellas reguladas por leyes diferentes, unas 300 -las primeras 10 son el 50\%-; subsistema privado: medicina prepaga -también regulado por leyes nacionales- y efectores -legislación concurrente-.

Por ello, sostenemos la necesidad de un acuerdo o ley convenio o marco, y legislación federal consecuente -no "nacional" como indican algunos autores ya que desde la génesis de su nombre podría indicar una centralización indeseada- de salud. No se trata vulnerar autonomías provinciales sino de definir el papel que corresponde a cada actor. La planificación y control de la ejecución de políticas públicas en los territorios debe contar con un Estado Nacional que conduzca las políticas sanitarias. La carencia de una ley que coordine la financiación y aseguramiento, y que establezca una cobertura universal, consecuencia de la descentralización (a nivel geográfico) y de la segmentación (entre subsistemas), acota las posibilidades de brindar una cobertura homogénea que garantice una verdadera equidad en la asignación de recursos de salud.

\section{Derecho de la salud y prestaciones de salud}

En el actual umbral de consideración social y jurídica de la salud, surge el vínculo entre equidad e igualdad, como consecuencia de una evolución que recoge el conjunto de valores abrazado por las sociedades democráticas modernas abrazaron a partir del siglo XVIII, en especial desde la Revolución Francesa. Bajo la forma de declaraciones de derechos, se consagró la igualdad en sentido formal, esto es, en el sentido de "igualdad ante la ley". Luego del auge de la Revolución Industrial -en realidad de la doble revolución como bien indica Hobsbawm (11) surge el Constitucionalismo Social (12) que llevó a concebir la igualdad como igualdad material plasmándose en las Constituciones y las leyes como "derechos de contenido material". Estos derechos, que pueden ser llamado de "prestación" en contraposición al Constitucionalismo Liberal que reconocía derechos de 
"abstención"- que gozan de total legitimidad, pero no están exentos de complicaciones en la práctica. En primer lugar, el ejercicio de los derechos requiere, como condición necesaria, que existan los medios materiales para financiarlos. En segundo lugar, se necesita que el derecho esté bien definido y que su contenido no tienda a generar un costo imposible de financiar. Este doble problema pone de manifiesto que, en busca de la equidad, no toda buena pretensión puede convertirse en derecho, y un derecho no puede tener contenidos infinitos, sin afectar la eficiencia.

En la Argentina, como sabemos, el Estado interviene sostenidamente desde mediados de la década de 1940, coexistiendo desde entonces tres modelos. Paradójicamente, en la década de 1990, de retroceso del Estado Nacional como prestador de oferta asistencial a través de sus propios efectores, surge el Programa Médico Obligatorio (PMO) con el Decreto 492/95. Este crea una Comisión Técnica que tiene como misión fundamental formular un programa médico asistencial, el cual consiste en un régimen de asistencia obligatoria para todas las Obras Sociales del Sistema Nacional del Seguro de Salud (leyes 23.660 y 23.661) las cuales tienen como fin asegurar a sus beneficiarios mediante servicios propios o contratados, prestaciones de prevención, diagnóstico y tratamiento médico-odontológico sin establecer períodos de carencia, ni coseguros o copagos fuera de lo expresamente indicado (13), (14).

EI PMO constituye una canasta básica de prestaciones que las Obras Sociales y las Empresas de Medicina Prepaga deben brindar obligatoriamente a sus beneficiarios y afiliados. El PMO incluye fundamentalmente atención primaria, plan materno-infantil, atención del recién nacido, cobertura materna durante el embarazo y el parto a partir del momento del diagnóstico y hasta el primer año de vida luego del nacimiento, atención del recién nacido hasta cumplir un año de edad, prevención del cáncer femenino, odontología preventiva, cobertura en enfermedades oncológicas, entre otras. A partir de la citada resolución que le dio origen, sufrió numerosas incorporaciones y modificaciones. Luego de la Resolución 939/00 del Ministerio de Salud se creó una Comisión Asesora integrada por representantes de los Agentes del Seguro y el Ministerio de Salud. La Resolución modifica integralmente el PMO con el objeto de definir con mayor claridad las prácticas incluidas, formulando un catálogo de prestaciones resultado de la revisión sistemática del Nomenclador Nacional. En el año 2002 y tras el Decreto 486/02 que declara la Emergencia 
Sanitaria, nace el Programa Médico Obligatorio de Emergencia (Resolución 201/02) con el objeto de establecer las prestaciones básicas esenciales necesarias e imprescindibles a raíz de la grave crisis que atravesaba el país en ese momento. Posteriormente se dicta la Resolución 1991/05 que integra al PMO las prestaciones establecidas en la Resolución $201 / 02$

El PMO constituye un estándar mínimo de protección, y no una enumeración taxativa. Es una canasta básica de prestaciones médico-asistenciales. La obra social debe brindar las prestaciones del PMO y otras coberturas obligatorias, sin carencias, preexistencias o exámenes de admisión. Mediante Resolución 939/2000, el Estado Nacional lo definió en sus considerandos como "un conjunto de servicios de carácter obligatorio como piso prestacional por debajo del cual ninguna persona debería ubicarse en ningún contexto".

De ello, se deduce que tal programa constituye un piso o una base, y por ello no debe constituir nunca fundamento a la negación de brindar la cobertura peticionada. Pues los prestadores de servicio de Salud deben adaptarse a las técnicas nuevas que puedan practicarse para procurar brindar el mejor sistema de salud integral.

Ahora bien, en los hechos el PMO pareciera adaptarse a los recursos, o mejor, a ciertas presiones sobre cómo administrarlos. Si el Estado pretende recuperar su protagonismo como generador y armonizador de las políticas públicas de salud, debe ser menos parcial. La idea de permanencia y sustentabilidad puede reconocer en los ciclos políticos ciertas improntas, pero lo que deben mandar son los determinantes, los objetivos y las metas para encararlos y mejorar la salud individual y colectiva. No habrá cobertura universal ni protección social en salud sin financiación pública y reglas claras, lo que se traduce en seguridad para el sistema. $\mathrm{Y}$ todo ello importa previsibilidad y mejores condiciones de acceso, evitando, por ejemplo, la judicialización de la salud vía amparos muchas veces necesaria para hacer cumplir el piso establecido a favor del ciudadano- pero a veces forzada en estrategia de extensión vertical de la cobertura, que genera dudas y amenazas sobre la sustentabilidad futura del sistema del sistema de salud. 


\section{La legislación sanitaria dictada por el Congreso de la Nación. Su aplicación en las provincias y municipios}

El sistema diseñado en la Constitución de 1853/1860, por influencia de la carta magna norteamericana, fue el del llamado "federalismo dual", es decir, la creación de dos espacios de poder perfectamente diferenciadas que presupone una estricta demarcación entre las competencias del Estado Nacional y de los Estados Provinciales. Por ello, y a partir de las complicaciones y fricciones que pudieran llegar a ocurrir en cuanto al ejercicio de competencias delegadas, reservadas y por supuesto concurrentes, es que se propugna la coordinación y la articulación entre las esferas de gobierno.

En materia de salud, se ha procurado lograr esa articulación en el Consejo Federal de Salud (COFESA), creado por Decreto 22.373 del año 1980, con propuestas para fortalecer su funcionamiento. Ratificado como instancia en los acuerdos de 2002 y 2003, es reforzado en 2009 al crearse el Consejo Federal Legislativo de Salud (COFELESA) integrado con los miembros de las comisiones de salud, o su equivalente, del Senado de la Nación, de la Cámara de Diputados de la Nación, de cada una de las legislaturas provinciales y de la Ciudad Autónoma de Buenos Aires. Es un organismo deliberativo de origen político cuyo objetivo es la articulación y promoción de políticas legislativas comunes en materia de salud en todo el territorio argentino. Entre sus funciones se resaltan la de armonizar y promocionar la aplicación de leyes comunes relativas a salud en todo el país, realizar el seguimiento y control de la aplicación de leyes relativas a salud, estudiar, asesorar y elaborar proyectos legislativos en materia de salud. El 3 de diciembre de 2014, se sancionó la ley 27.054, mediante la cual el Senado y la Cámara de Diputados de la Nación aprobaron el Pacto Federal Legislativo de Salud.

La Nación Argentina se encuentra organizada como estado federal con cuatro niveles diferenciados de gobierno: Nación, provincias, Ciudad Autónoma de Buenos Aires y municipios. Esto se traduce en un sistema jurídico multinivel y plurilegislativo en el cual coexisten diversos ordenamientos jurídicos, emergentes del ejercicio de potestades normativas propias de cada uno de los sujetos de la relación federal (14). Por su parte, los municipios luego de la reforma constitucional de 1994 han sido reconocidos como autónomos en los órdenes institucionales, políticos, administrativos y económicofinancieros, pero como bien lo ha interpretado la jurisprudencia de la Corte Nacional, 
especialmente en el caso "Ponce" (15) esta autonomía debe ser parte de un sistema institucional orientado hacia la descentralización y fundado en un federalismo cooperativo. En este sentido, son las provincias las encargadas de diseñar los alcances y contenidos de la autonomía municipal.

Así, en lo relacionado con el Derecho Sanitario, como en tantas otras ramas jurídicas, se destaca la presencia del estado multinivel y plurilegislativo, lo que deviene necesariamente en la coexistencia en un mismo territorio de cuatro niveles de gobierno. Es aquí donde torna relevante la concepción de "federalismo de concertación" esgrimida por nuestro entrañable maestro Pedro J. Frías.

Pero debemos regresar a los profundos cambios estructurales que impactaron e el sector de la salud en la década del noventa. Cobró importancia la descentralización y desconcentración (Nación-Provincias-Municipios), procesos cuyo objetivo fue establecer una canasta básica de prestaciones y la orientación de los recursos del Estado hacia la satisfacción de esa base en los sectores más vulnerables. Se decía que se alcanzarían mejores niveles de eficiencia y calidad en los servicios, y aumentar la satisfacción de los usuarios. Hubo un generalizado proceso de transferencia de efectores y del recurso humano afectado de la Nación a las provincias, y en muchos casos de éstas hacia los municipios, muy traumático en su implementación. Se traspasaron los hospitales, pero hubo dificultades con la reasignación de los recursos necesarios. No se tuvo en cuenta la desigualdad entre provincias (infraestructura, recursos humanos, entre otros) ya que la provincialización del sistema de salud vino de la mano del desfinanciamiento estatal al sector, lo que, en un sistema de salud fragmentado, desarticulado y sin adecuada coordinación interjurisdiccional atentó contra la vigencia del derecho de la salud, tanto en el acceso como en la calidad del servicio.

Entonces, en un Estado Federal, ¿en qué consisten los deberes del Estado Nacional? Siguiendo a Clérico (16): a) aprobar leyes u otras normas para el efectivo ejercicio de los derechos (caso del PMO o algunas de las leyes mencionadas anteriormente; b) disponer y organizar la forma en que se deben realizar esos derechos, fiscalizar y coordinar con las otras jurisdicciones las acciones que se deban realizar y establecer procedimientos para que los derechos sean exigibles en caso de incumplimiento. c) Intervenir y colaborar para disminuir las desigualdades entre las provincias. Tiene un deber de coordinación de los 
servicios asistenciales de acuerdo a la organización federal de nuestro país; d) prestación directa de servicios de salud.

El gobierno nacional y los gobiernos provinciales y municipales deben definir y consensuar un eje articulador de las reformas, que apunte a mejorar la situación de los ciudadanos sin cobertura explícita, hacer más eficiente la prestación de salud y mejorar la equidad del sistema. Sin embargo, no debe impulsarse la aplicación de políticas homogéneas en todas las regiones, sino que por el contrario debe reconocerse la necesidad de articular sistemas diferentes y de brindar soluciones flexibles, reconociendo una estructura federal e intentando aprovechar las fortalezas de cada jurisdicción. Se trata de comprender las dificultades de cada Provincia, sus capacidades y sus preferencias con la idea de construir un grupo de instancias en las cuales se pueda construir paulatinamente una modalidad flexible de organización federal.

\section{Competencias Provincia - Nación}

La salud es un bien constitucionalmente protegido, por el artículo 75, además de lo estipulado en el artículo 33 y en el artículo 42 de nuestra Constitución. Sumado a ello, el Estado Nacional es el responsable del cumplimiento de las obligaciones internacionales contraídas en esta materia. El Estado Nacional aparece entonces como el último garante del cumplimiento de estas obligaciones. Como veíamos anteriormente la Corte ha establecido que el Estado Nacional es la autoridad rectora en las políticas de salud y el garante de este derecho "el Estado no puede desligarse del deber de promover y facilitar las prestaciones de salud que requiera la comunidad so pretexto de la inactividad de otras entidades públicas o privadas". En el fallo "Asociación Benghalensis" (17) la Corte estableció que las provincias son parte integrante del sistema y resultan indispensables para una eficiente implementación de la normativa vigente. A su vez destacó, que el derecho a la salud no sólo se encuentra contemplado en la Constitución Nacional sino también en las provinciales, por lo que se infiere que las acciones destinadas a su protección constituían una responsabilidad compartida con las provincias, dejando en claro que se trata de una competencia concurrente, ya que se trata de intereses comunes, por lo que se hace imprescindible el establecimiento de una adecuada coordinación de funciones entre los distintos niveles de gobierno. 
Básicamente y para sintetizar encontramos que el Ministerio de Salud es la máxima autoridad nacional en materia de salud, compartiendo con otras instancias del gobierno nacional el área de programas sociales. Entre otras competencias, corresponde a este Ministerio la conducción del sector en su conjunto a través del diseño de programas, dictado de normas y ejecución de acciones que permitan la coordinación entre los distintos subsectores. Sin embargo, dada la estructura federal del país, los gobiernos provinciales cuentan con total autonomía en materia de políticas de salud, lo que hace que los lineamentos del nivel nacional tengan solamente un valor indicativo, estando la adhesión a los mismos condicionada a las coincidencias en el plano político más general. A su vez, los municipios tienen a cargo la ejecución de programas y la administración de los servicios de su órbita. La mala combinación entre federalismo y descentralización resultó en desigualdades en términos geográficos y sociales, con una diversidad de normas y de ofertas de servicios. Existe un abanico normativo y una gran diversidad de oferta de servicios que se suman a la multiplicidad de instituciones de las obras sociales y del sector privado. A su vez, la naturaleza federal determina que los Ministerios de Salud de cada provincia sean responsables tanto de la política sanitaria de su jurisdicción como del presupuesto público para sustentarla, con limitada injerencia de la autoridad nacional. Recordemos que el COFESA no es una estructura formalmente vinculante. Los acuerdos que se alcanzan en esa instancia se tornan relevantes para los Ministerios provinciales en la medida en que existe consenso político acerca de las líneas de acción y recursos nacionales que las sustenten. Por ello, son los Ministerios de Salud de las provincias las instituciones de mayor relevancia en el diseño de políticas y en la definición de los mecanismos que organizan y reforman los sistemas de salud de sus jurisdicciones.

\section{Legislación nacional de orden público y de adhesión}

La Nación ejerce la práctica legislativa mediante la sanción de leyes en materia de salud utilizando dos fórmulas principales: a) orden público; b) Invitación a adherir a las provincias (en la mayoría de los casos declarando de interés nacional algún tipo de tratamiento o cobertura).

El concepto de "orden público" y el de "interés nacional" presentan dificultades a la hora de intentar explicarlos y de ubicar su esfera de acción. La noción de "orden público" 
resulta muchas veces un verdadero enigma. La doctrina ha expuesto múltiples criterios para conceptualizar el orden público. Para resumir, encontramos dos criterios: el mayoritario que lo concibe como:

un conjunto de principios eminentes -religiosos, morales, políticos y económicos- a los cuales se vincula la digna subsistencia de la organización social establecida (18)

y el minoritario que sostiene que

"una cuestión es de orden público cuando responde a un interés general, colectivo, por oposición a la cuestión de orden privado, en la cual sólo juega un interés particular" (18)

Más allá, que como señala Borda (18), definir el orden público ha desesperado a muchos jurisconsultos, entendemos como orden público cuando el interés general se encuentra comprometido y merece ser protegido para garantizar que siempre prevalezca sobre los intereses particulares, y cuyo contenido es variable por principios que pueden mutar según evolucione la sociedad y el sistema político de un país. De ahí que puede considerarse algo de orden público en un momento y lugar y puede más tarde no merecer esa clasificación. Las leyes que utilizan la fórmula de orden público lo hacen para que la ley tenga validez en todo el territorio nacional y generalmente se encuentra en el primer artículo o en los últimos. Pero ¿hasta qué punto las provincias deben acatar lo establecido por la Nación en materias que son de competencia concurrente? ¿Las leyes de orden público obligan a los Estados Provinciales o sólo a los particulares? En nuestro país se ha difundido un concepto desacertado y de rasgos centralistas, que supone que cualquier ley del Congreso es superior y obligatoria para los gobiernos provinciales. Ello importa desconocer la autonomía provincial -argucia atribuida a Mitre para explicar la subordinación aún en aquellos temas que las provincias no atribuyeron competencia a la Nación, manteniendo su soberanía sobre ellos-, sin respetar el reparto de materias reservadas y delegadas que surge de la Constitución Nacional. En las primeras, en modo alguno puede prevalecer una legislación provincial o federal, puesto que ello implicaría la violación de la Constitución Nacional, en virtud del art. 31. Las Provincias actúan de manera autónoma dentro de los ámbitos de competencia que les fija la Constitución. En el caso de competencias concurrentes, es lógico que existan leyes de orden público que rijan 
en todo el territorio nacional pues es necesario para cumplimentar objetivos sanitarios a fin de implementar políticas, evitando los conflictos entre las diversas jurisdicciones y resguardando, ante todo, los derechos de los ciudadanos.

Por otra parte, encontramos la fórmula de Adhesión, en la que se invita a las provincias a adherir, generalmente en el último artículo de la norma. El federalismo de concertación se ha plasmado a través justamente de las leyes federales de adhesión. Aquí ya no se trata de una cooperación basada en un acuerdo (como en el caso de las leyes convenio) sino de la adhesión provincial a un régimen creado unilateralmente por el gobierno federal. Generalmente se trata de leyes que declaran de "interés nacional" algún tipo de tratamiento o cobertura. Ahora bien, a qué se refiere el concepto de interés nacional: ¿Se trata de "interés estatal”? ¿Exige algún compromiso al Estado Federal? ¿Al Gobierno de la República? ¿Implica algún tipo de situación de excepción, como por ejemplo una emergencia sanitaria, o de señalamiento jurídico institucional específico, como por ejemplo el que cabe dentro del ancho mundo del "interés público"? ¿O se trata de una declaración de interés típica de las que el Congreso o las Legislaturas tratan a menudo en espectro que va desde una feria hasta un congreso o un festival? (19). Buscando en la legislación encontramos la clasificación de declaración de interés nacional en leyes que establecen la cobertura de tratamiento médicos (cobertura para autistas, celíacos, entre otros), y en otras que reconocen un emprendimiento cultural, que protegen una especie de arbusto, que celebran la realización de una muestra agropecuaria, o hasta aquellas que catalogan como recurso estratégico la energía eólica. Básicamente la categoría de interés nacional se parece más a un "mercado persa" donde uno puede elegir a qué actividad asignarlo sin importar la relevancia que le asista, que a un concepto estrictamente jurídicolegislativo.

Las leyes de adhesión se basan en facultades concurrentes existentes que posibilitan que las provincias reconozcan, faciliten, amplíen, promuevan, desarrollen, los derechos reconocidos por la normativa federal. Entonces, no se trata de que la legislación federal inhiba las facultades provinciales. Siempre los gobiernos locales pueden, a través de sus constituciones o legislación ampliar, superar y mejorar el ejercicio de los derechos ya previstos. Hay otra versión de adhesión, que consiste en que la ley nacional invita a la provincia a dictar normas similares, por ejemplo la ley de obesidad 26.396, contra la cual 
las provincias optaron por seguir esa iniciativa, o bien adhirieron a la norma, con cierta imprecisión porque la adhesión no importa una "clonación" de normas provinciales como reflejo de las nacionales (ejemplo: si la norma nacional crea un programa nacional de lucha contra la enfermedad, la adhesión no implica la creación de un programa provincial).

Estas fórmulas nacionales erráticas, que llegan al mundo político, institucional, jurídico y sanitario en particular sin aparente planificación o estrategia alguna, teniendo en cuenta el peso de la provincia en la regulación de efectores y ejercicio central del poder de policía sanitario combinado con la fragmentación del sistema de salud, ha provocado un régimen cuasi anárquico, desarticulado, que en cuestiones básicas de protección de derechos provoca una clara distorsión en cuanto al acceso y a la equidad.(3).

\section{Conclusiones}

1. Destacamos la consolidación del "derecho a la salud" -facultad-como expresamente tutelado por la Constitución, la vigencia de un cuerpo de normas específicas destinadas a su abordaje -dentro del derecho común y también en un cuerpo específico de "legislación" o "derecho objetivo sanitario", y el estudio del Derecho Sanitario como cuerpo científico jurídico o disciplina autónoma. El derecho sanitario supone un campo de actividades sociales delimitado y propio, dentro del cual reciben aplicación sus normas particulares. La legislación sanitaria constituye un cuerpo coherente de normas que regulan las distintas relaciones jurídicas en el ámbito sanitario. Esta rama requiere de la interdisciplina y del diálogo otras ramas ya existentes, para afrontar los problemas de fragmentación y segmentación del sistema de salud argentino, expresando la plena actualidad y vigencia de su necesidad.

2. Hemos reconocido un Estado plurilegislativo y multinivel; y a pesar de que el proceso de que nuestro régimen federal se ha desnaturalizado a medida que en la práctica fiscal ha avanzado un fuerte centralismo, en la realidad operativa sanitaria se ha acentuado la fragmentación.

3. La "Legislación Sanitaria" emana de 25 jurisdicciones -la Nación, la Ciudad Autónoma de Buenos Aires y las 23 Provincias-, y a su vez la cobertura de la salud se encuentra distribuida entre el sector público, las Obras Sociales y el sector privado, con 
una pobre coordinación entre subsectores, que ofrecen prestaciones en salud muy heterogéneas.

4. El PMO que define las prestaciones a ser cubiertas rige sobre obras sociales nacionales y prepagas, es decir, involucra solo a la mitad de los argentinos (20). A su vez, la idea de un PMO es dinámica, y supone muchas veces agregar coberturas que deben contemplar la necesaria actualización de costos frente a la permanente incorporación de nuevas obligaciones prestacionales, lo que en la práctica no ha acontecido de modo regular.

5. Los distintos prestadores muchas veces no pueden dar respuesta a los diferentes requerimientos en el caso concreto por lo que se plantean conflictos judiciales generados ante los innumerables requerimientos de prestaciones de salud, no todas incluidas en el PMO. Lo que no se resuelve en sede administrativa se dirime en sede judicial.

6. El nuevo Código Civil al receptar la constitucionalización del derecho privado genera que el Derecho a la Salud adquiera protagonismo como derecho humano y fundamental.

Gradualmente se está abriendo una nueva perspectiva para el derecho sanitario. Actualmente encontramos una legislación sanitaria que a pesar de enfocarse en la persona atraviesa dificultades. Celebramos las leyes que se enfocan en el individuo y su relación con el campo de la salud, pero por una parte, es necesario prevenir contra una manipulación de esa facultad, así como también contra una proliferación de "leyes remedio" o "leyes terapia" que implican un exagerado esfuerzo del legislador para nominar o poner en el centro de la escena alguna patología en particular cuando su abordaje quizá puede ser mejor contemplado por acciones e incluso instrumentos normativos más abarcativos. Por otra parte, es necesario establecer mecanismos concretos para su financiamiento. Las prestaciones deben incluir la manera en que se van a financiar ¿Hasta dónde soportará el PMO? ¿Hasta dónde soportará el sistema con reglas inestables e incompletas que quizá sin buscarlo terminan alentando la judicialización de la salud?

A su vez, la fragmentación del sistema provoca inequidad y desigualdad en el acceso. Asimismo, las competencias de los distintos niveles de gobierno para legislar en salud y su ejecución no estandarizada termina provocando desigualdades (por un lado) y mayor peso en algunas jurisdicciones más sólidas en su oferta (por otra). No renegamos de la distribución de competencias propio de nuestro sistema federal, pero la dificultad de 
coordinar la legislación deviene en complicaciones propias de la falta de consenso y articulación. Aún el COFESA y el COFELESA no han dado respuesta suficiente. Es por ello que necesitamos vencer inercias e intereses encontrados. Debemos renovar el federalismo en materia de salud a partir de un desarrollo integral que incorpore al Gobierno Nacional, a las provincias y a los municipios en la decisión y ejecución de las políticas sanitarias. A través de una o varias leyes federales de salud, el estado Nacional ejercería una verdadera rectoría y también una coordinación interjurisdiccional.

\section{Referências}

1.Organización Mundial de la Salud - OMS-. Disponible en http://www.who.int/governance/eb/who constitution sp.pdf. [Acceso: 2 mayo 2017].

2.Organización Panamericana de la Salud -OPS-. Disponible en http://www1.paho.org/spanish/HPP/OttawaCharterSp.pdf [Acceso: 2 mayo 2017].

3.Martínez PF. La construcción del mundo jurídico multidimensional. Córdoba: Advocatus; 2003.

4.Ciuro C. Á. El derecho de la salud en el complejo jurídico. Cadernos Ibero-Americanos de Direito Sanitário, 2012, 1 (1): 8-29. Disponible en: http://www.cadernos.prodisa.fiocruz.br/index.php/cadernos/article/view/36/81 [Acceso 2 mayo 2017]

5.Lalonde, M. El concepto de "campo de salud": una perspectiva canadiense. En Promoción de la salud: una antología. Organización Panamericana de la Salud. Publicación Científica. (557); 1996: 3-5.

6.Frutos, J, Royo, MA. Salud pública y epidemiología. España: Diaz de Santos; 2006.

7.Argentina. Corte Suprema de Justicia de la Nación. Tolosa Nora Elida y otro c/Swiss Medical S.A. 27 de agosto de 2013.

8.Argentina. Corte Suprema de Justicia de la Nación. Los saladeristas Podestá, Bertram, Anderson, Ferrer y otros contra la Provincia de Buenos Aires; sobre indemnización de daños y perjuicios. 14 de mayo de 1887.

9.Garay, O. La legislación sanitaria y el derecho a la salud. La Ley. 2012.

10. Argentina. Corte Suprema de Justicia de la Nación. Saguir y Dib, Claudia Graciela s/ autorización, 06 de noviembre de 1980

11.Hobsbawm, E. La Era de la Revolución 1789-1848, La Era del Capital 1848-1875, La

Era del Imperio 1875-1914. Barcelona: Crítica; 2012. 
12. De la Fuente, H. Los jueces y las leyes de orden público, La Ley; 2004.

13. Argentina. Corte Suprema de Justicia de la Nación. Hospital Británico de Buenos Aires c/ Estado Nacional; Ministerio de Salud y Acción Social, 13 de marzo de 2001;

14. Argentina. Corte Suprema de Justicia de la Nación. Asociación de Esclerosis Múltiple de Salta c/ Ministerio de Salud - Estado Nacional s/ acción de amparo - medida cautelar, 18 de diciembre de 2003; Argentina. Corte Suprema de Justicia de la Nación.

Floreancig, Andrea Cristina y otro por sí y en representación de su hijo menor H., L. E. c/ Estado Nacional s/ amparo, 11 de julio de 2006; entre otros.

15.Argentina. Corte Suprema de Justicia de la Nación. Ponce, Carlos Alberto c/ San Luis, Provincia de s/acción declarativa de certeza, 24 de febrero de 2005.

16.Clérico, L. El derecho a la salud de las personas con discapacidad ¿ El argumento del federalismo como acelerador o como freno? La Ley, Suplemento Derecho Constitucional. 2009.

17.Argentina. Corte Suprema de Justicia de la Nación. Asociación Benghalensis y otros c. Estado nacional, 01 de junio de 2000.

18.Borda, G. Concepto de ley de orden público, La Ley. 1950

19.Cetrágonlo, O. Financiamiento fragmentado, cobertura desigual y falta de equidad en el sistema de salud argentino. Revista de Economía Política de Buenos Aires, 13 (8).

Disponible en: http://bibliotecadigital.econ.uba.ar/download/ecopoli/ecopoli v8 n13 05.pdf. [Acceso 2 mayo 2017]

20.Anlló, GC. O. Políticas sociales en Argentina: viejos problemas, nuevos desafíos, Crisis, Recuperación y Nuevos Dilemas. La Economía Argentina 2002- 2007. Santiago de Chile: Kosacoff - Comisión Económica para América Latina y el Caribe (CEPAL).; 2007. Disponible en:

http://repositorio.cepal.org/bitstream/handle/11362/28481/S2007021 es.pdf;jsessionid=DA 7904302A9663121318BD7 4EBB51540?sequence=1 [Acceso 2 mayo 2017] 